\title{
Estudantes com altas habilidades ou superdotação: desdobramentos dos índices da Sinopse Estatística e dos Microdados na Região Sudeste do Brasil
}

\author{
Students with high abilities or giftedness: developments in the Synopsis and Microdata indexes in \\ the Southeast Region of Brazil \\ Estudiantes con altas capacidades o superdotación: evolución de los índices de Sinopsis Estadística \\ y Microdatos en la Región Sudeste de Brasil
}

Recebido: 13/03/2021 | Revisado: 20/03/2021 | Aceito: 25/03/2021 | Publicado: 29/03/2021

\author{
Rosemeire de Araújo Rangni \\ ORCID: https://orcid.org/0000-0002-8752-9745 \\ Universidade Federal de São Carlos, Brasil \\ E-mail: rose.rangni@ufscar.br \\ Carlos Samuel Rossi \\ ORCID: https://orcid.org/0000-0002-1749-9114 \\ Universidade Federal de São Carlos, Brasil \\ E-mail: carlossamuelrossi@estudante.ufscar.br \\ Fabiana Oliveira Koga \\ ORCID: https://orcid.org/0000-0002-4646-1537 \\ Faculdade de Ensino Superior do Interior Paulista, Brasil \\ E-mail: fabianapsicopedagogiamusical@gmail.com
}

\begin{abstract}
Resumo
As altas habilidades ou superdotação (AHSD) pertence ao público da Educação Especial e os estudantes têm direito ao atendimento educacional especializado, garantido na legislação brasileira. Nessa perspectiva, eles são cadastrados pelas escolas e esses registros são armazenados, analisados e publicizados pela Sinopse Estatística e Microdados. O problema de pesquisa parte dos dados da Sinopse Estatística e dos Microdados da Região Sudeste do Brasil, nos anos de 2018 e 2019, ambos disponibilizados pelo Instituto Nacional de Estudos e Pesquisas Educacionais Anísio Teixeira (INEP), vinculado ao Ministério da Educação. A Região Sudeste, brasileira, é composta por quatro Estados: Espirito Santo, Minas Gerais, Rio de Janeiro e São Paulo. Para tanto, o objetivo foi investigar e analisar os índices de matrículas de estudantes com AHSD da Sinopse Estatística e dos Microdados da Região Sudeste do Brasil nos anos de 2018 e 2019. O método utilizado foi a Pesquisa Documental de caráter qualitativo e quantitativo. A coleta de dados foi por meio das buscas na Sinopse Estatística e Microdados e analisadas em sete variáveis elencadas, nas quais foram aplicadas a tabela cruzada e a frequência. Os resultados obtidos indicaram uma diferença nos dados da Sinopse Estatística em comparação ao Microdados, pois, nos anos de 2018 e 2019 a Sinopse Estatística apresentou um aumento de $486,42 \%$ de estudantes com AHSD e nos Microdados houve um crescimento de 387,22\% no mesmo período. Conclui-se que, os Microdados requerem mais transparência de acesso e exploração dos dados.
\end{abstract}

Palavras-chave: Altas habilidades ou superdotação; Sinopse estatística; Microdados; Região sudeste.

\begin{abstract}
High skills or giftedness (AHSD) belongs to the Special Education public and the students have right to specialized educational service, guaranteed in the Brazilian law. In this perspective, they are registered by the schools, and these records are stored, analyzed, and published by the Statistical Synopsis and Microdata. The research problem starts from the data of the Statistical Synopsis and Microdata of the Southeast Region of Brazil, in the years 2018 and 2019, both made available by the National Institute of Educational Studies and Research Anísio Teixeira (INEP), both made available by the Ministry of Education. The Brazilian Southeast Region is composed of four states: Espirito Santo, Minas Gerais, Rio de Janeiro, and São Paulo. Therefore, the objective was to investigate and analyze the enrollment rates of students with AHSD from the Statistical Synopsis and Microdata of the Southeast Region of Brazil in the years 2018 and 2019. The method used was qualitative and quantitative Documentary Research. Data collection was through the searches in the Statistical Synopsis and Microdata and analyzed in seven listed variables, in which the cross table and frequency were applied. The results obtained indicated a difference in the data from the Statistical Synopsis compared to the Microdata, because in the years 2018 and 2019 the Statistical Synopsis showed an increase of $486,42 \%$ of students with AHSD, and in the Microdata there was a growth of $387,22 \%$ in the same period. It is concluded that Microdata requires more transparency of access and exploration of data.
\end{abstract}

Keywords: High abilities or giftedness; Statistical synopsis; Microdata; Southeastern region. 


\begin{abstract}
Resumen
Altas capacidades o superdotación (AHSD) pertenecen al público de Educación Especial y los estudiantes tienen derecho a asistencia educativa especializada, garantizada por la ley brasileña. Desde este punto de vista, las escuelas los registran y estos registros son almacenados, analizados y publicados por la Sinopsis Estadísticas y los Microdatos. El problema de investigación se basa en los datos de la Sinopsis Estadística y los Microdatos de la Región Sudeste del Brasil, en los años 2018 y 2019, ambos puestos a disposición por el Instituto Nacional de Estudios e Investigaciones Educativas Anísio Teixeira (INEP), vinculado al Ministerio de Educación. La Región Sudeste de Brasil está compuesta por cuatro Estados: Espírito Santo, Minas Gerais, Río de Janeiro y São Paulo. Para ello, el objetivo fue investigar y analizar las tasas de matrículas de estudiantes con AHSD de la Sinopsis Estadística y los Microdatos de la Región Sudeste del Brasil en los años 2018 y 2019. El método cualitativo y cuantitativo utilizado para la Investigación Documental. La recogida de datos se realizó mediante búsquedas en la Sinopsis Estadística y Microdatos y se analizó en siete variables listadas, en las que se aplicó la tabla cruzada y la frecuencia. Los resultados obtenidos indicaron una diferencia en los datos de la Sinopsis Estadística comparados a los Microdatos, ya que en los años 2018 y 2019 la Sinopsis Estadística mostró un incremento del $486,42 \%$ de estudiantes con AHSD y en los Microdatos hubo un crecimiento del $387,22 \%$ en el mismo período. Se concluye que, los Microdatos requieren más transparencia de acceso y exploración de los datos.
\end{abstract}

Palabras clave: Altas capacidades o superdotación; Sinopsis estadística; Microdatos; Región sudeste.

\title{
1. Introdução
}

As altas habilidades ou superdotação ${ }^{1}$ (AHSD), neste artigo, serão discorridas brevemente sob a perspectiva teórica de Renzulli (2021), na qual traça a existência de dois tipos de superdotação (termo empregado pelo autor traduzido do inglês giftedness). O primeiro tipo denominado de Superdotação Escolar se caracteriza por destaque em disciplinas escolares, sendo o mais fácil de identificar por testes de Quociente de Inteligência (Q.I) e de desempenho. O segundo tipo é a Superdotação Criativo-produtiva e, está relacionado à criatividade, à capacidade investigativa e à originalidade.

Em sua Teoria dos Três Anéis, Renzulli $(2014$;2021) definiu o comportamento superdotado na interação de três conjuntos, representados em círculos: habilidade acima da média, criatividade e comprometimento com a tarefa e ele não se restringe somente aos três conjuntos, pois, há influência da personalidade e de fatores ambientais externos, chamada de Houndstooth Background, diagramada como uma malha xadrez atrás dos três anéis, conforme ilustra a Figura 1).

Figura 1 - Houndstooth Background - A concepção de superdotação.

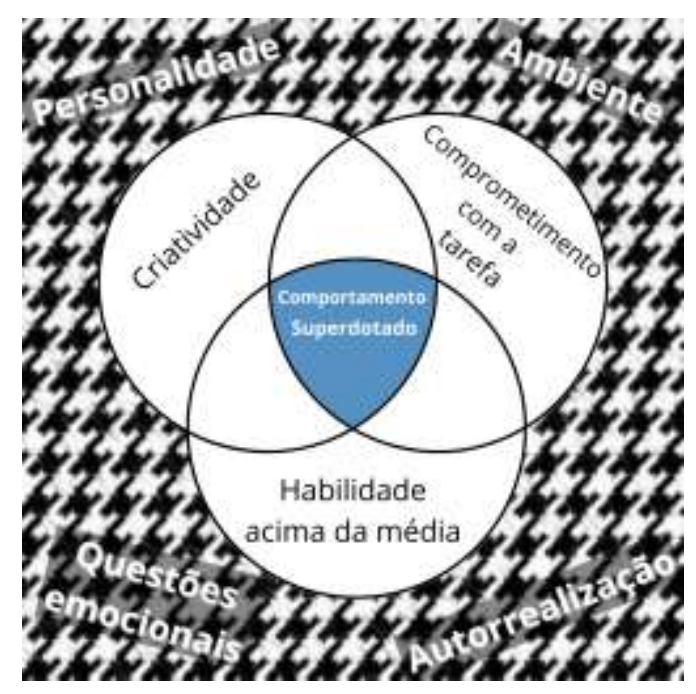

Fonte: Autores, Adaptado de Renzulli (2018, p. 24).

\footnotetext{
${ }^{1}$ Será empregada a terminologia de acordo com a Lei de Diretrizes e Bases da Educação Nacional (LDBEN) (Brasil, 1996), entretanto, serão respeitados os termos originais dos autores.
} 
O primeiro anel representa a Habilidade Acima da Média e está dividida em duas partes: Habilidade Geral e Habilidade Específica. A Habilidade Geral é aquela que pode ser aplicada em quase todas as áreas de conhecimento, as quais consistem na capacidade de processar informações e de integrar experiências, que resultem em respostas apropriadas e adaptáveis a novas situações e na capacidade de se envolver em pensamentos abstratos, espaciais, verbais, memória e numérica. A Habilidade Específica trata da capacidade de adquirir conhecimento, habilidade ou capacidade de realizar uma ou mais atividades de um tipo específico com rapidez. O Comprometimento com a Tarefa está intimamente relacionado a fatores da personalidade e às áreas de interesse do indivíduo e a motivação que irá desenvolver habilidades criativas. Já o conjunto da Criatividade envolve características como originalidade de pensamento e resolução de problemas, pensamento divergente, compreensão da forma visual, planejamento, consciência humana e propostas sociais (Renzulli, 2014; 2021).

Nesse sentido, a sensibilidade do olhar para estas características é importante tendo em vista que os testes psicométricos não os contemplam para a identificação das AHSD. É nessa perspectiva que Renzulli (2018) traz a contribuição do Modelo de Enriquecimento Escolar (SEM) para desenvolver a superdotação criativo-produtiva mediante às características ambientais, que influenciam de forma direta na potencialização de habilidades específicas. Sob a ótica do autor, ao desenvolver esse modelo, o sistema educacional, bem como os educadores, poderão proporcionar aos alunos o máximo de oportunidades, a alta realização, a satisfação e o bem-estar por meio do enriquecimento curricular do mais geral ao mais específico, relacionados com fatores ambientais, personalidade, com atividades exploratórias gerais e de treinamento em grupo e investigações de problemas reais de forma individual ou grupal. Essas habilidades desenvolvidas estão relacionadas às características criativo-produtiva e de liderança, sendo recomendável que os testes psicológicos não sejam a única possibilidade para identificação dos estudantes.

Sobre a existência de sujeitos com AHSD, Renzulli (2021) afirma estarem entre 10\% a 15\% de qualquer população, pois é sugerível que os órgãos competentes os identifiquem e os cadastrem (Brasil, 1996), a fim de proporcionar a criação de políticas públicas. A definição de AHSD considerada oficialmente, no Brasil, é:

III $-[\ldots]$ aqueles que apresentam um potencial elevado e grande envolvimento com as áreas do conhecimento humano, isoladas ou combinadas: intelectual, liderança, psicomotora, artes e criatividade (Brasil, 2009 p.17).

Por essa razão, o censo escolar tem sido uma fonte importante de dados estatísticos para o Ministério da Educação (MEC) e as demais instâncias.

Os dados estatísticos (censos) possibilitam, de acordo com Field (2009) e Koche (2011), analisar fenômenos oriundos da realidade, a fim de conhecê-los empiricamente. Acrescenta, ainda, que em um universo grande de dados contribui para a análise, reflexão e tomada de decisão frente a um determinado comportamento populacional e a probabilidade de representação alta, utilizando-se de modelos estatísticos é eficaz. Um exemplo disso é o trabalho realizado pelo Instituto Nacional de Estudos e Pesquisas Educacionais Anísio Teixeira (Inep)².

O Inep está vinculado ao Ministério da Educação (MEC) e é responsável pelas avaliações, exames e tabulações estatísticas, possibilitando ao governo acompanhar o desempenho da Educação Básica e outras modalidades de ensino, assim, cria e viabiliza políticas públicas e recursos com base nos índices das avaliações nacionais. Entre os dados produzidos pelo Inep estão a Sinopse Estatística e os Microdados.

A Sinopse Estatística reúne dados referentes à matrícula, função docente, rendimento escolar em diferentes modalidades como: Educação Básica, Educação Especial, Educação de Jovens e Adultos e a Educação Superior. Os dados são

\footnotetext{
${ }^{2}$ http://inep.gov.br/web/guest/sobre-o-inep
} 
disponibilizados por regiões e por unidades da federação. Eles são divulgados oficialmente e são tabulados e organizados por uma equipe técnica especializada (Resende \& Lacerda, 2013, Inep, 2020).

Por outro lado, os Microdados são constituídos por um nível menor de desagregação, de acordo com o Inep (2020), embora seja distinto da Sinopse eles reúnem a mesma natureza de dados, porém sem uma tabulação ou tratamento estatístico prévio. As informações dos Microdados são obtidas via download e podem ser lidas e tabuladas por softwares como: SAS $^{3}$ e SPSS $^{4}$. Para a leitura dos dados é recomendável estabelecer um percurso de procedimentos de verificação mediante cálculos e/ou cruzamentos de variáveis, o que não acontece com na Sinopse Estatística, isso porque o cálculo já está pronto. É possível inferir que, os Microdados são mais livres que a Sinopse ao possibilitar o leitor realizar a sua própria análise, utilizando os dados disponíveis no banco oferecido pelo Inep (Ibid, 2020; Moreira \& Carvalho, 2014).

Diante dos dados estatísticos oriundos do supracitado banco e a criação de políticas públicas, inclusive para a Educação Especial, o MEC pode organizar a distribuição de recursos, cuja origem é do Fundo de Manutenção e Desenvolvimento da Educação Básica (FUNDEB). De acordo com Rebelo e Kassar (2018) o FUNDEB consiste em um fundo financeiro voltado para as escolas públicas em geral para custear os gastos educacionais e de recursos, inclusive, aqueles destinados à Educação Especial. Esse fundo foi criado com base na Constituição Federal de 1988, a qual determina que Estados, Municípios e o Distrito Federal devem aplicar 25\% da receita de seus impostos arrecadados para financiamento da Educação pública. No entanto, recentemente esse percentual foi votado e discutido, ficando em $20 \%$ o repasse da alíquota dos impostos arrecadados (Senado Federal, 2020).

Em decorrência dessas ações realizadas pelo Inep, em consonância com a legislação federal, cada Estado possui autonomia para gerir suas bases legais pertinentes ao atendimento às AHSD, público da Educação Especial (Brasil, 1996).

É preciso destacar que, o Brasil tem extensa dimensão territorial com população estimada de 212.643.704, em 2020, de acordo com o Instituto Brasileiro de Geografia e Estatística (IBGE) ${ }^{5}$. A pesquisa em tela foi direcionada à Região Sudeste do Brasil, por ser a mais populosa. Ela é composta de quatro Estados: Espirito Santo (ES), com população estimada em 4.064.052 pessoas, Minas Gerais (MG), com 21.292.666, Rio de Janeiro (RJ), com 17.366.189 e São Paulo (SP), com 46.289.333, perfazendo um total populacional de 89.012.234.

Para além, de acordo com o IBGE, na Educação Básica o Estado do Espírito Santo possui Índice da Educação Básica $(\text { IDEB })^{6}$ de 5,7 nos anos iniciais do ensino fundamental ( $8^{\circ}$ lugar entre os 26 Estados e o Distrito Federal no ranking nacional) e nos anos finais do ensino fundamental é avaliado com o índice de 4,4 (12 lugar dentre os 26 Estados e o Distrito Federal), sendo que seu Índice de Desenvolvimento Humano (IDH) ${ }^{7}$ é de 0,740 , ocupando a $7^{a}$ posição quando comparado com os 26 Estados e o Distrito Federal.

O Estado do Rio de Janeiro pontua IDEB de 5,3 nos anos iniciais do ensino fundamental ( $16^{\circ}$ lugar entre os 26 Estados e o Distrito Federal no ranking nacional) e nos anos finais do ensino fundamental têm índice de 4,2 (16 lugar entre os 26 Estados e o Distrito Federal). Além disso, o IDH do Estado é de 0,761 em $4^{a}$ posição quando comparado com dos 26 Estados e o Distrito Federal.

\footnotetext{
${ }^{3}$ https://www.sas.com/pt_br/home.html

${ }^{4}$ https://www.ibm.com/br-pt/products/spss-statistics

${ }^{5}$ https://www.ibge.gov.br/

${ }^{6}$ Índice de Desenvolvimento da Educação Básica formulado pelo Inep, em 2007, para medir a qualidade do aprendizado em território nacional, a fim de estabelecer metas para a melhoria do ensino em todo Brasil. http://portal.mec.gov.br/conheca-o-ideb

7 Índice de Desenvolvimento Humano (IDH) criado para comparar indicadores de países nos itens riqueza, alfabetização, educação, esperança de vida, natalidade, entre outros, tendo como principal objetivo avaliar o bem-estar da população com atenção especial nas crianças. https://www.ipea.gov.br/desafios/index.php?option=com_content\&view=article\&id=2144:catid=28\&Itemid=23
} 
Minas Gerais tem 6,3 nos anos iniciais do ensino fundamental, ocupando $2^{\circ}$ lugar entre os 26 Estados e o Distrito Federal no ranking nacional, e nos anos finais do ensino fundamental é de 4,5, $10^{\circ}$ lugar entre os 26 Estados e o Distrito Federal. O IDH desse Estado é de 0,731 e está na 9a posição, comparado aos outros entes federados.

Por fim, São Paulo tem índice de 6,5 nos anos iniciais do ensino fundamental, em primeiro lugar no ranking nacional, e, nos anos finais do ensino fundamental possui índice de 4,9, indo para a terceira colocação entre os 26 Estados e o Distrito Federal. O IDH de São Paulo é de 0,783, estando em segunda posição.

Para compor o estudo, investigou-se sobre as legislações de cada Estado da Região Sudeste, disponíveis nos portais das secretarias de educação, que pudessem referir-se ao público das AHSD. O Gráfico 1 ilustra esses resultados.

Gráfico 1 - Legislações vigentes nos Estados da Região Sudeste.

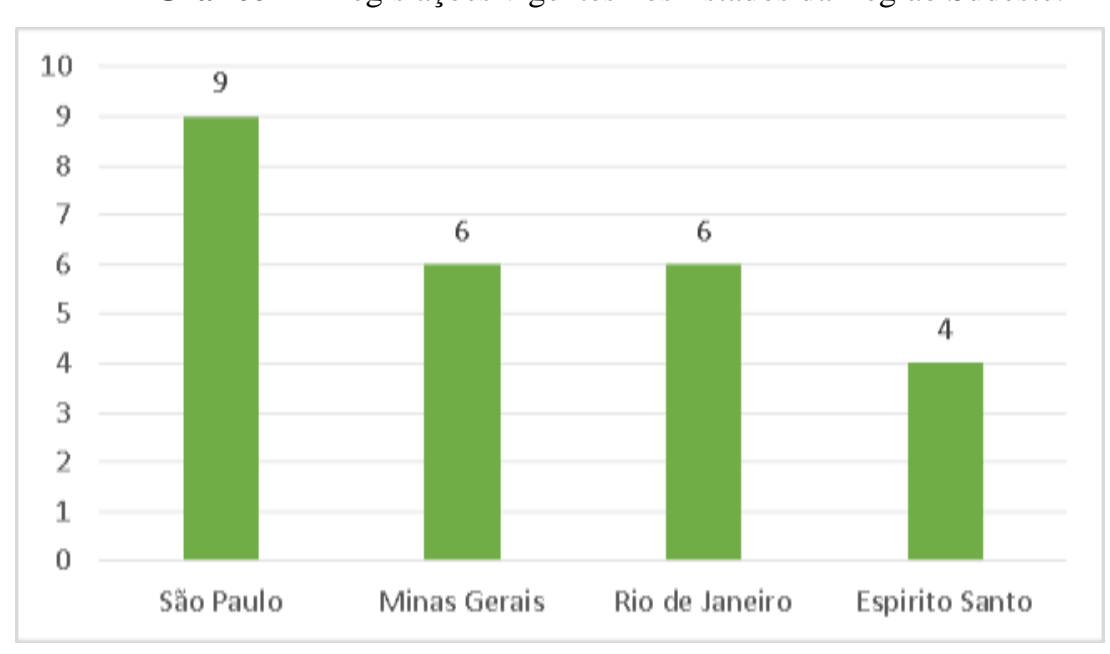

Fonte: Autores.

É possível observar a quantidade de legislação existente no Estado de São Paulo voltada para a Educação Especial e que considera as AHSD.

Com base no índice total de legislações encontradas da Região Sudeste $(n=25)$ e a existência do censo escolar houve buscas de artigos que explorassem as Sinopses Estatísticas e os Microdados como abordagem principal de pesquisa e que tratassem das AHSD, concomitantemente. Foram encontradas pouquíssimas produções.

Sobre a Sinopse Estatística, nos últimos dez (10) anos, algumas pesquisas apareceram inclusive na área de Educação Especial, porém, em AHSD somente foi encontrada a produção de Oliveira e Rangni (2019) e as demais na área de deficiência e de transtornos. Em referência às produções relacionadas aos Microdados não houve achados voltados para as AHSD.

Ademais, ao analisar o aumento de matrículas de estudantes com AHSD em classes comuns na Região Sudeste entre 2018 (n=6.406) para 2019 (n=31.212), considerando a Sinopse foi constituída a questão de pesquisa entre a Sinopse Estatística e os Microdados, qual seja: Quais os índices de matriculas de estudantes com AHSD na Educação Básica na Sinopse Estatística e nos Microdados da Região Sudeste em 2018 e 2019? Para respondê-la, instaurou-se como objetivo investigar e analisar os índices de matriculas de estudantes com AHSD da Sinopse Estatística e dos Microdados da Região Sudeste do Brasil nos anos de 2018 e 2019.

Os dados serão discutidos embasados em: Brasil (1996), Colozio, et al. (2021), Macalli et al. (2018), Melletti (2014), Renzulli (2021, 2018, 2014), Resende e Lacerda (2013), Sá e Cia (2015), Sales e Silva (2013), Santos e Elias (2018) e Silva et al. (2020), bem como as informações fornecidas publicamente pelo Governo Federal e os Governos Estaduais. 


\section{Metodologia}

O estudo trata-se de uma Pesquisa Documental, restringindo-se à coleta de dados voltada para documentos, que podem ser arquivos públicos, publicações parlamentares, censos, estatísticas, entre outros. Também, valeu-se de pesquisa qualitativa e quantitativa com o estudo de relações de variáveis. Esse tipo de pesquisa volta-se para a descoberta de variáveis no que tange à determinada questão ou situação, fornecendo dados para a verificação de uma hipótese (Marconi \& Lakatos, 2011; Pereira et al., 2018; Koche, 2011).

A coleta dos dados ocorreu por meio dos portais referente à Sinopse Estatística (Banco 1) e ao Microdados (Banco 2), ambos oriundos do site do Inep ${ }^{8}$. Para tanto, selecionou-se o recorte temporal de 2018 e 2019, com foco na Região Sudeste.

Para manter o grau de confiabilidade da tabulação, duas máquinas/computadores realizaram os cálculos no software SPSS. Foi seguida a sequência de variáveis e, ao final, confrontaram-se os valores para verificar se alcançaram os mesmos resultados ou não. Esses resultados eram acessados a partir de um relatório extraído em formato PDF gerado pelo Software SPSS.

Para a leitura e compreensão dos resultados o portal da Sinopse Estatística traz o sumário e notas explicativas, localizadas abaixo dos resultados. O banco dos Microdados conta com um dicionário e tabelas auxiliares, os quais trazem os significados de cada código, sendo preciso ressaltar que no SPSS somente números são permitidos, pois há a necessidade de criação de rótulos para as variáveis que serão calculadas. Tanto as explicações da Sinopse quanto do dicionário e das tabelas auxiliares do Microdados estão em formato Excel. A Figura 2 representa o percurso do método empregado para a pesquisa.

Figura 2 - Representação das etapas do método.

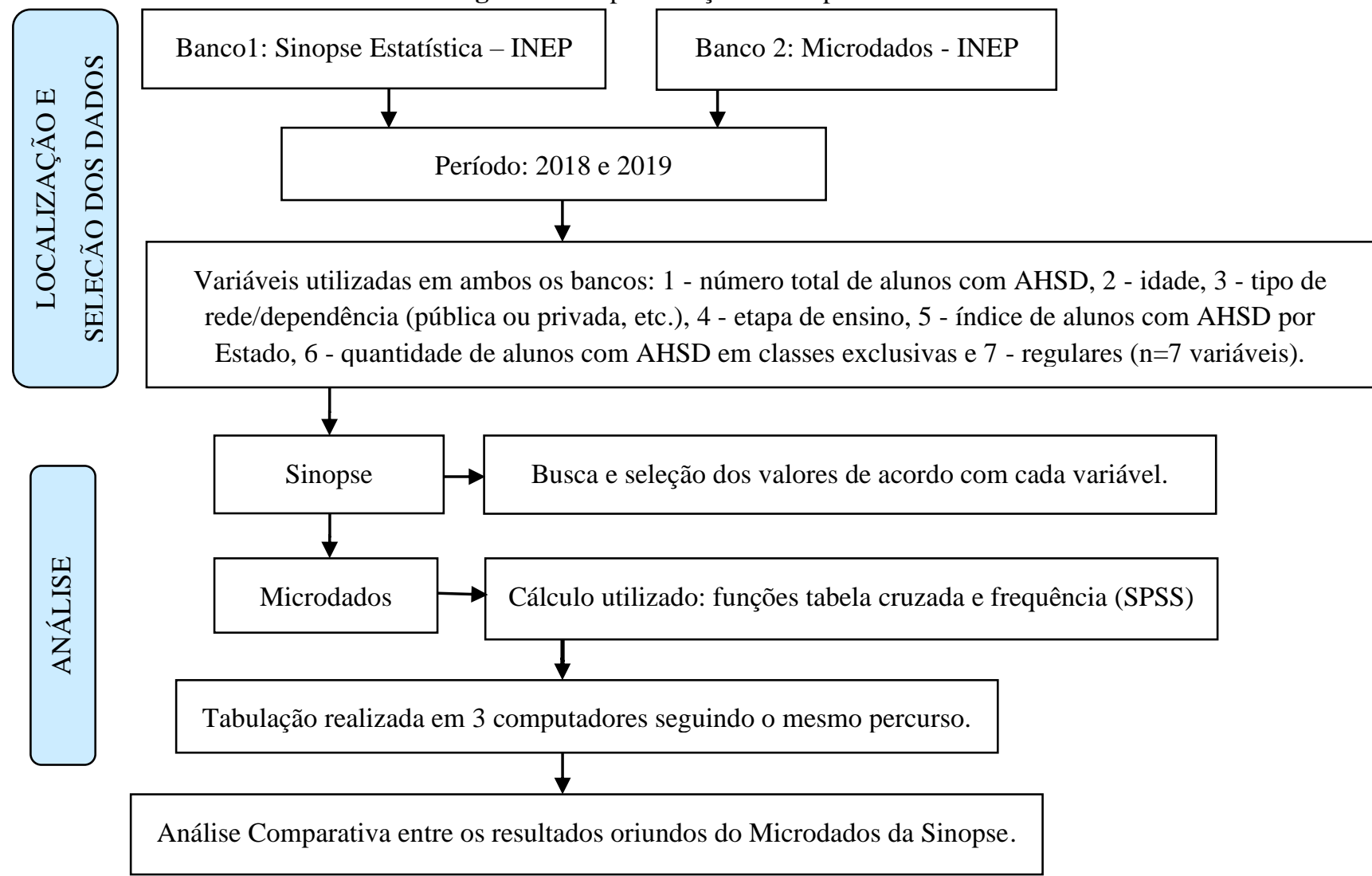

Fonte: Autores, de Prisma diagramas. http://prisma-statement.org/prismastatement/flowdiagram.

\footnotetext{
${ }^{8}$ http://inep.gov.br/web/guest/dados. Há páginas específicas para o acesso de cada um dos bancos de dados.
} 
As variáveis foram selecionadas com base no que apresentavam os portais da Sinopse Estatística e Microdados no percurso de seleção de Sales e Silva (2013) e Melletti (2014). Na tabulação, utilizando a função frequência, foi possível encontrar o resultado total de matrículas, em que as variáveis nu_ano_censo e ID_matrículas foram combinadas. As demais tabulações foram realizadas com base no cruzamento das variáveis, cujas ocorrências deram-se da seguinte maneira: (1) Número total de alunos com AHSD - variáveis nu_ano_censo na linha ${ }^{9}$ e in_superdotação na coluna no SPSS; (2) Idade nu_ano_censo e in_superdotação na linha e nu_idade na coluna combinadas; (3) Tipo de rede (publica, privada)/Dependência - nu_ano_censo e in_superdotação na linha e tp_dependência na coluna (cruzadas); (4) Etapa de ensino - nu_ano_censo e in_superdotação na linha e tp_etapa_ensino na coluna; (5) Número (código) por Estado - nu_ano_censo e in_superdotação na linha e CO_UF na coluna; com relação às (6) classes exclusivas foram realizados dois cruzamentos: in_especial_exclusiva (coluna) e in_superdotação (na linha); e (7) classes regulares: in_regular (na coluna) e in_superdotação (na linha). A análise dos dados coletados seguiu a seguinte ordem: a) Sinopse Estatística e b) Microdados.

\section{Resultados}

Os resultados foram analisados de acordo com as variáveis elencadas e conforme apresentados a seguir.

\section{a) Sinopse Estatística}

A Tabela 1 expõe os dados encontrados nos anos de 2018 e 2019.

Tabela 1 - Total de estudantes com e sem AHSD na Sinopse Estatística da Região Sudeste - 2018 e 2019.

\begin{tabular}{|c|c|c|c|c|c|}
\hline \multirow{6}{*}{ Sinopse } & Estado & 2018 & Classes Comuns & Classes Exclusivas & Total \\
\hline & SP & & 1.929 & 11 & 1.940 \\
\hline & RJ & & 1.250 & 9 & 1.259 \\
\hline & ES & & 1.582 & 0 & 1.582 \\
\hline & $\begin{array}{l}\text { MG } \\
\text { Total }\end{array}$ & & 1.645 & 64 & 1.709 \\
\hline & Sudeste & & 6.406 & 84 & \\
\hline \multirow{6}{*}{ Sinopse } & Estado & 2019 & Classes Comuns & Classes Exclusivas & Total \\
\hline & SP & & 26.616 & 6.022 & 32.638 \\
\hline & RJ & & 1.356 & 8 & 1.364 \\
\hline & ES & & 1.549 & 0 & 1.549 \\
\hline & MG & & 1.691 & 45 & 1.736 \\
\hline & $\begin{array}{l}\text { Total } \\
\text { Sudeste }\end{array}$ & & 31.212 & 6.075 & \\
\hline
\end{tabular}

Fonte: Resultados extraídos das Sinopses Estatísticas na íntegra.

Pode-se observar o aumento considerável de matrículas de estudantes com AHSD na Região Sudeste, a olhos vistos, entre 2018 e 2019, também, nota-se discrepância entre os Estados com expressividade no Estado de São Paulo.

As Figuras 3 e 4 ilustram as matrículas por Estado em classes comuns (CC) e em classes exclusivas (CE), nos anos de 2018 e 2019.

\footnotetext{
${ }^{9}$ Linhas e colunas são campos para o preenchimento no SPSS para que as variáveis possam ser calculadas.
} 
Figura 3 - Região Sudeste: total de estudantes com altas habilidades ou superdotação em Classes Comuns (CC) e Classes Exclusivas (CE), por Estado nas Sinopses Estatísticas em 2018.

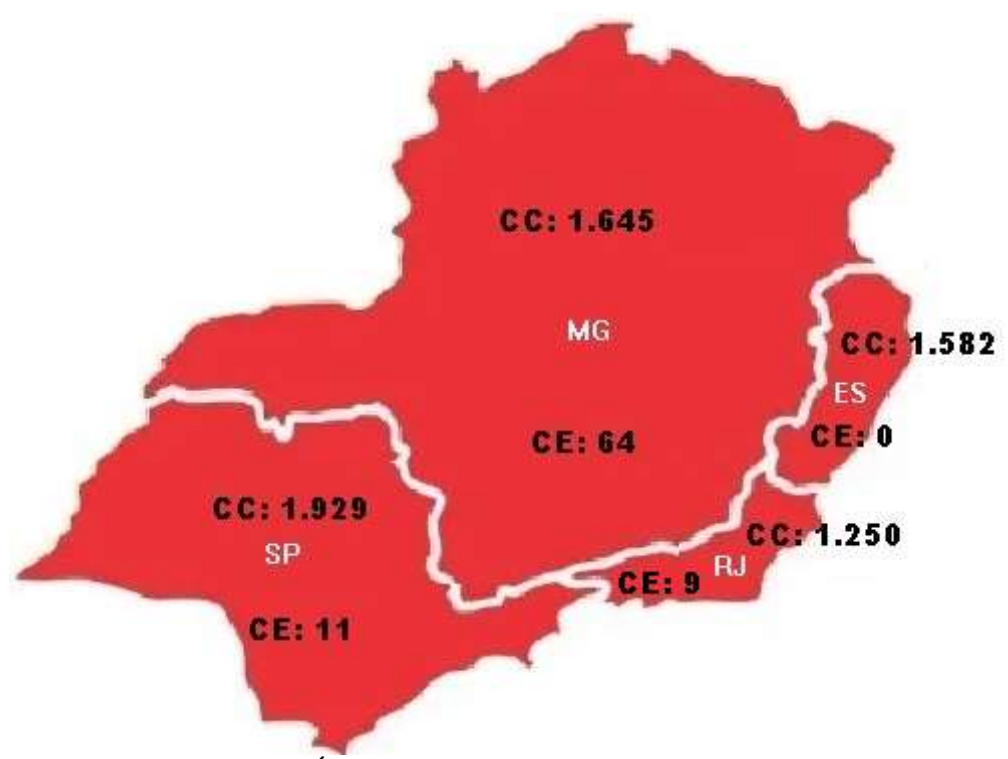

Fonte: Autores. Índices extraídos da Sinopse Estatística.

Preliminarmente é possível notar que o Estado de São Paulo concentra o maior número de matrículas e o Estado do Rio de Janeiro o menor no ano de 2018 e que a maioria dos estudantes está inserida em classes comuns. A figura 4 apresenta o total de estudantes com AHSD no ano de 2019.

Figura 4 - Mapa da Região Sudeste com o total por Estado de estudantes com AHSD em Classes Comuns (CC) e Classes Exclusivas (CE) por Estado na Sinopse Estatística em 2019.

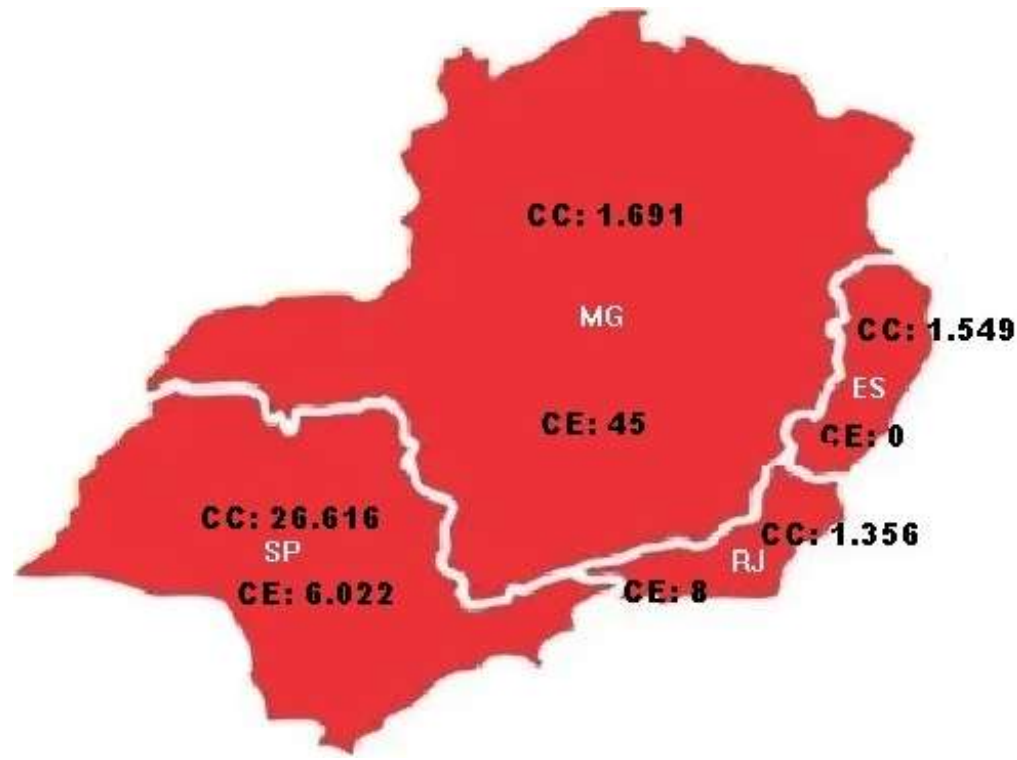

Fonte: Resultados extraídos da Sinopse Estatística na íntegra.

Diante dos índices de 2019, percebe-se que houve um crescimento do número de matrículas em classes comuns nos Estados da Região Sudeste, mais consideravelmente no Estado de São Paulo com um aumento de $1.280 \% 10$ em classes comuns.

$1026.616 \times 100 / 1.929-100=1.279,78=1.280 \%$; Prova real: $1.929+1.279,78 \%=26.616$. 


\section{b) Microdados}

Neste item foram expostos os resultados encontrados no portal do Microdados mediante as variáveis selecionadas para o estudo, as quais são: (1) Número total de alunos com AHSD; (2) Idade; (3) Tipo de rede (publica, privada) / Dependência; (4) Etapa de ensino; (5) Número (código) por Estado; (6) classes exclusivas e (7) classes regulares.

(1) Número total de alunos com AHSD

Por meio dos relatórios gerados pelo sistema SPSS, o total de estudantes com AHSD em 2018 e 2019 estão exemplificados na Tabela 2 .

Tabela 2 - Microdados: Estudantes com e sem AHSD nos censos de 2018 e 2019.

\begin{tabular}{cccc}
\hline Ano & $\begin{array}{c}\text { Estudantes com altas } \\
\text { habilidades ou superdotação }\end{array}$ & $\begin{array}{c}\text { Estudantes sem altas } \\
\text { habilidades ou superdotação }\end{array}$ & $\begin{array}{c}\text { Total de } \\
\text { matriculas }\end{array}$ \\
\hline 2018 & 8.808 & 20.149 .245 & 20.158 .053 \\
2019 & 51.652 & 19.733 .193 & 19.784 .845 \\
\hline Total & 60.460 & 39.882 .438 & 39.942 .898 \\
\hline
\end{tabular}

Fonte: MEC/INEP. Censo Escolar da Educação Básica: Microdados (2018, 2019).

Ao analisar os dados é preciso destacar o índice de estudantes com AHSD em relação aos estudantes sem AHSD. Outro ponto saliente é o aumento expressivo de estudantes com AHSD na Região Sudeste entre 2018 e 2019.

(2) Idade

Quanto à variável idade pode-se inferir que o número de identificação de estudantes com AHSD concentra-se, acentuadamente, no período do Ensino Fundamental, em fase inicial, entre seis (6) e nove (9) anos. O Gráfico 2 ilustra a distribuição. 
Gráfico 2 - Estudantes com AHSD por idade.

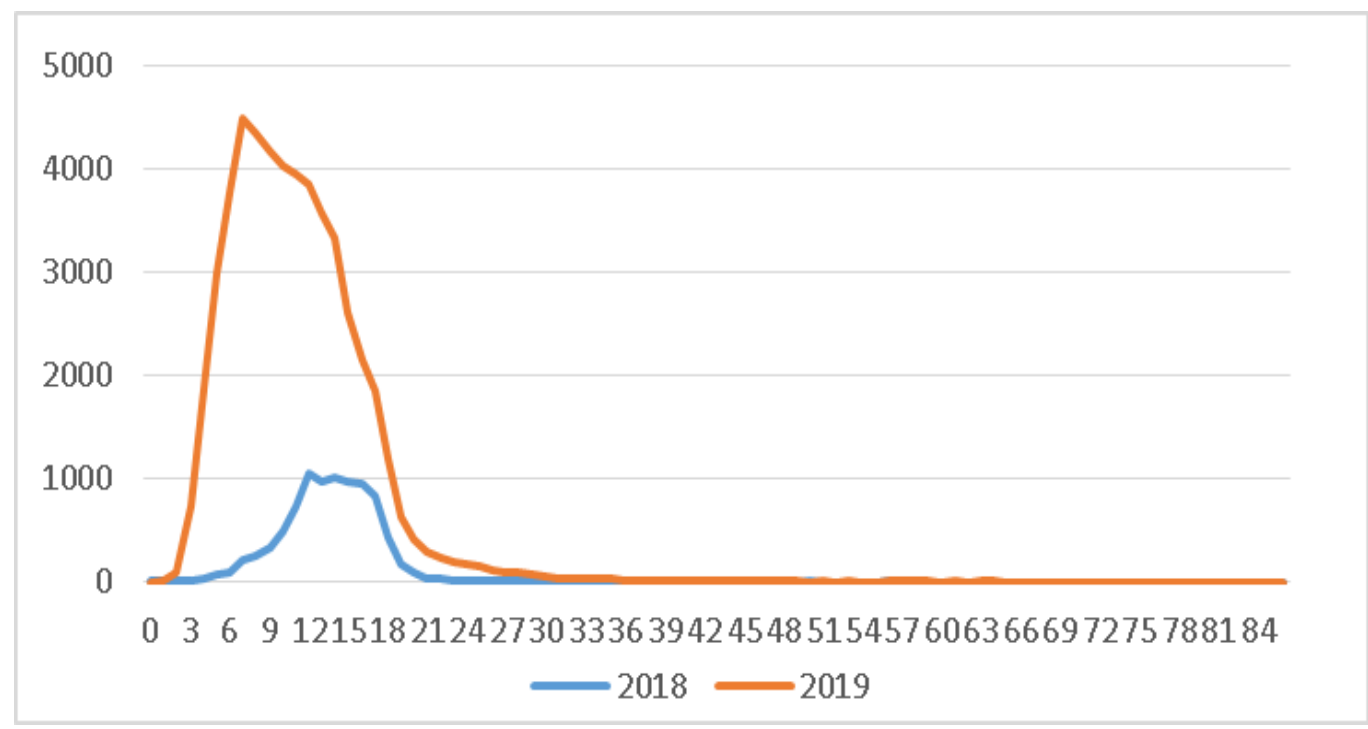

Legenda: n=8808 pessoas com altas habilidades ou superdotação em 2018 e n=51652 em 2019. Fonte: MEC/INEP. Censo Escolar da Educação Básica: Microdados (2018, 2019).

De um lado há de se mencionar que no relatório emitido pelo SPSS, após a análise, a partir de 84 anos não seguiram a sequência numérica ordinal, o que limitou a exemplificação no Gráfico 2. Por outro lado, não havia estudantes com AHSD entre as idades de 84 a 95 anos, esta era a idade máxima constante no relatório do SPSS. As Figuras 5 e 6 exemplificam o erro sequencial ocorrido no relatório de 2018 e 2019.

Figura 5 e 6 - Demonstração do erro ocorrido na sequência da apresentação dos resultados referentes à idade.
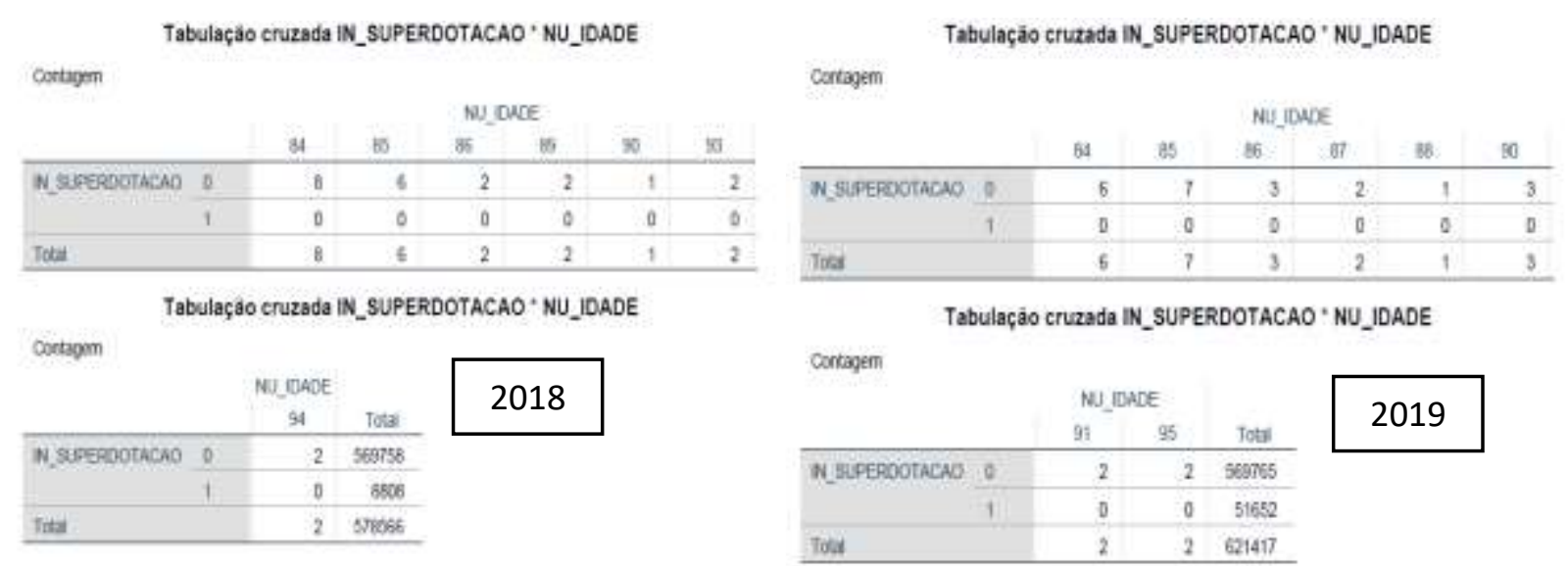

Fonte: Resultados extraídos na íntegra dos relatórios gerados pelo SPSS

Independentemente de haver estudantes com AHSD da terceira idade, entende-se que não é recomendável apresentar esse tipo de equívoco no sistema.

(3) Tipo de rede (publica, privada) / Dependência

A Tabela 3 apresenta a tabulação da variável dependência. Em uma análise preliminar pode-se notar o maior índice de matrículas de estudantes com AHSD na rede municipal de ensino. 
Tabela 3 - Microdados: Resultado da distribuição de estudantes com AHSD por dependência (2018-2019).

\begin{tabular}{lcc}
\hline \multicolumn{1}{r}{ Dependência } & $\mathbf{2 0 1 8}$ & $\mathbf{2 0 1 9}$ \\
\hline Federal & 264 & 289 \\
Estadual & 3.320 & 12.604 \\
Municipal & 4.171 & 27.841 \\
Privada & 1.053 & 10.918 \\
\hline Total & 8.808 & 51.652 \\
\hline
\end{tabular}

Fonte: MEC/INEP. Censo Escolar da Educação Básica.

Possivelmente, as matrículas estejam relacionadas às iniciativas e ações de cada dependência e, talvez, à formação de educadores voltada para Educação Especial e às AHSD, o que pode ter colaborado para incentivar a identificação dos estudantes.

\section{(4) Etapa de ensino}

Durante a tabulação dos dados referentes à etapa de ensino foi detectado um equívoco no relatório gerado pelo SPSS. Ele apresentou os índices de modo discriminado das etapas de ensino, porém a somatória dessas etapas relacionada aos estudantes com AHSD não coincidiu aos índices de 2018 e de 2019, bem como o montante correspondente aos estudantes sem AHSD. Por exemplo, em 2018 deveria aparecer $n=8.808$, mas na tabela consta $n=6.490$, fruto da somatória dos valores de estudantes com AHSD nas etapas de ensino, sendo que o mesmo ocorreu em 2019. Dessa forma, deveria ser na somatória geral $\mathrm{n}=51.652$, mas na tabela consta $\mathrm{n}=37.287$. A seguir as Figuras 7 e 8 confirmam o equívoco.

Figura 7 e 8 - Microdados: variável etapas de ensino na tabulação dos dados de 2018 e 2019.

\begin{tabular}{|c|c|c|c|c|c|c|c|c|c|c|c|c|c|}
\hline \multirow{2}{*}{ sen } & \multicolumn{6}{|c|}{ PP_ETAPA_ESSND } & \multicolumn{6}{|c|}{ TP_ETAPA_ESSNO } & \multirow[b]{2}{*}{ Total } \\
\hline & & 68 & 70 & 71 & 73 & 74 & Tstal & & & 71 & 73 & 74 & \\
\hline \multirow[t]{2}{*}{ N_SJPERDOTACAO } & 0 & 12310 & 10099 & 5619 & 4 & 31 & 41-1927 & \multirow[t]{2}{*}{ IN SUPERDOTACAO } & 0 & 5581 & 185 & 39 & 41262 \\
\hline & 1 & 20 & 57 & 83 & 0 & 2 & 6490 & & 1 & 183 & 3 & 2 & 37287 \\
\hline \multicolumn{2}{|l|}{ Total } & 12230 & 16156 & 5702 & 4 & 38 & 421417 & \multicolumn{2}{|l|}{ Total } & 5764 & 188 & 41 & 449529 \\
\hline
\end{tabular}

0 - não superdotados; 1- superdotados. Fonte: Autores. Resultados extraídos na íntegra dos relatórios gerados pelo SPSS.

Sobre as figuras 7 e 8 é preciso mencionar que cada etapa de ensino é representada por um número para que o SPSS possa tabular os resultados. Devido ao valor equivocado não foi possível considerar os resultados de cada etapa, isoladamente. (5) Número (código) por Estado

A tabulação realizada envolveu o índice de estudantes com AHSD em cada Estado pertencente à Região Sudeste, exposto na Tabela 4 . 
Tabela 4 - Microdados: distribuição de estudantes com AHSD (2018 - 2019).

\begin{tabular}{|c|c|c|c|}
\hline Estado & Código & $\begin{array}{l}\text { Índice de estudantes AHSD em } \\
2018\end{array}$ & $\begin{array}{l}\text { Índice de estudantes AHSD em } \\
2019\end{array}$ \\
\hline Minas Gerais & 31 & 2.710 & 2.602 \\
\hline Espírito Santo & 32 & 2.032 & 1.985 \\
\hline Rio de Janeiro & 33 & 1.697 & 1.854 \\
\hline São Paulo & 35 & 2.369 & 45.211 \\
\hline \multicolumn{2}{|c|}{ Total } & 8.808 & 51.652 \\
\hline
\end{tabular}

Fonte: MEC/INEP. Censo Escolar da Educação Básica: Microdados.

Os códigos dos Estados originam-se dos fornecidos pelo IBGE. Ao analisar a Tabela 4, em 2018, o Estado com o maior número de estudantes com AHSD foi Minas Gerais ( $\mathrm{n}=2.710)$, porém, em 2019, São Paulo apresentou (n=45.211). Houve um decréscimo no Estado de Minas Gerais e um aumento expressivo em São Paulo entre o biênio 2018 e 2019. Os decréscimos e aumentos podem ser percebidos nos outros dois Estados.

(6) classes exclusivas e (7) classes regulares

Os dados referentes às classes exclusivas e regulares para o atendimento das AHSD serão apresentados na Tabela 5.

Tabela 5 - Microdados: distribuição de estudantes com AHSD nas classes exclusivas e regulares (2018-2019)

\begin{tabular}{cccc}
\hline & $\mathbf{2 0 1 8}$ & $\mathbf{2 0 1 9}$ & Total \\
\hline $\begin{array}{c}\text { Classes } \\
\text { exclusivas }\end{array}$ & 84 & 6.075 & 6.159 \\
Classes regulares & 6.130 & 36.639 & 42.769 \\
$\begin{array}{c}\text { AEE } \\
\text { Enriquecimento } \\
\text { curricular }\end{array}$ & - & 9.172 & 9.172 \\
\hline Total & 6.214 & 51.886 & 58.100 \\
\hline
\end{tabular}

Fonte: MEC/INEP. Censo Escolar da Educação Básica: Microdados

Nota-se, que em relação ao índice total de estudantes atendidos com AHSD em classes exclusivas, classes regulares, AEE e enriquecimento curricular em 2018 e 2019, pelos cálculos dos Microdados, demonstra a concentração maior de estudantes em classes regulares, entretanto, isso pode não significar que eles estejam no AEE. Em 2018 o banco Microdados não apresentou os resultados de AEE devido à inexistência dessa variável no ano citado. Há uma ambiguidade nos resultados totais de educandos com AHSD em 2019. Mesmo que eles estejam distribuídos em classes exclusivas, classes regulares e em AEE_enriquecimento_curricular, a somatória total de estudantes com AHSD deveria ser n=51.652 (2019) e não n=51.886; representado pela porcentagem de $0,45 \%{ }^{11}$ de diferença no mesmo banco e ano.

Em 2018, na tabela 5, não foi possível fazer o mesmo cálculo devido à falta de acesso ao dado que só pode ser gerado por meio da existência da variável AEE_enriquecimento_curricular.

Assim, é perceptível que os estudantes com AHSD estão em classes regulares conforme previsto pela legislação, no entanto, o atendimento ainda é inferior ao que é visível no número total de matrículas e, também, há incertezas a respeito de algumas variáveis e tabulações. Desta forma, não foi possível saber a lógica que envolve o lançamento dos resultados do

${ }^{11}$ Diferença para mais do $n=51.652$ equivale a $n=234$ estudantes com AHSD. 
Censo, como também não é informado pelo INEP questões sobre a tabulação dos Microdados, tanto pelos softwares SPSS ou SAS, para obtenção de alguns valores relacionados ao número total de matrículas, os alunos que estão no AEE, em classes exclusivas, entre outros, para exemplificar. É visível, sobretudo, que não se tem conhecimento da margem de erro na leitura dos dados por meio do sistema SPSS e pelo próprio carregamento dos dados importados do INEP. Isso será aprofundado na discussão.

\section{Discussão e Considerações Finais}

Com o baixo índice de pesquisas interessadas em explorar os bancos de dados do INEP, em especial a Sinopse Estatística e Microdados, em relação às AHSD, torna-se um grande desafio discutir os dados com poucos interlocutores. Porém, aqueles encontrados apresentaram aspectos relevantes para a presente pesquisa: a dificuldade de criação e efetividade de políticas públicas voltadas para a inclusão, o AEE de estudantes com AHSD na Educação Básica em todo o Brasil (Silva, et al., 2020), a distribuição equitativa das verbas públicas para a Educação e a garantia de oportunidade para estudantes de diferentes setores da sociedade, principalmente, os com AHSD em situação de vulnerabilidade social e econômica (Colozio, et al., 2021; França, 2015; Sá \& Cia, 2015).

Os dados da Sinopse Estatística foram o ponto inicial para a inquietação respaldada nas diferenças significativas com os dados brutos dos Microdados. Todos os anos a Sinopse é divulgada e os Microdados são disponibilizados, porém a diferença está no acesso com domínio das ferramentas aos dados. Vale ressaltar que foi necessário consultar outros sites governamentais para buscar informações, bem como o dicionário disponibilizado pelo INEP para a leitura interpretativa do Microdados. Assim, foi possível cruzar as variáveis de forma a compará-las e quando divergiam ao estabelecer correspondência com as da Sinopse foi identificada a discrepância da totalidade de matrículas entre ambos os bancos de dados.

Essas diferenças chamaram atenção porque enquanto na Sinopse Estatística, em 2018, nas classes comuns, os alunos com AHSD correspondia a 0,034\% dos alunos matriculados na educação básica na Região Sudeste, no Microdados, no mesmo período, correspondia a 0,044\%. Já em 2019, na Sinopse os alunos com AHSD correspondia a 0,166\% dos alunos matriculados e no Microdados, no mesmo período, correspondia a $0,262 \%$, ou seja, um aumento de $387,22 \%$, de um ano para outro na Sinopse e $486,42 \%^{12}$ no Microdados. A diferença do aumento de alunos com AHSD matriculados na educação básica em classes comuns na Região Sudeste entre os dois bancos de dados corresponde cerca de 99,2\% em apenas um ano.

A diferença expressiva nos resultados da Sinopse Estatística e dos Microdados levantou a problemática, porque notase a diferença entre eles, a Sinopse Estatística sintetiza os resultados do censo escolar e sua análise, possivelmente, é realizada com a colaboração de técnicos do INEP. Infere-se que há uma estrutura analítica constituída, previamente, para ajudar a análise e organização desses dados e, além disso, há um objetivo traçado sobre quais variáveis seriam interessantes para vir a público.

Ao ler os Microdados torna-se necessário realizar análises preliminares utilizando-se do SPSS, pois cada usuário o acessa de diferentes maneiras porque não há instruções ou critérios sobre as combinações e uso das variáveis. Já na Sinopse isso não ocorre porque tudo já está tabulado e pronto para a leitura. Também, ao averiguar as pesquisas que utilizaram os Microdados destaca-se a pesquisa de Santos e Elias (2018), em que explicita o uso e combinações das variáveis.

Diante do mencionado, essa diferença na abordagem entre os bancos pode instaurar uma espécie de viés, inviabilizando o total controle das variáveis e suas combinações. Por isso, Macalli et al. (2018) recomendam o conhecimento estatístico antes de apreender sobre os Microdados. Na pesquisa de Melletti (2014) observou-se que cada pesquisador pode seguir um determinado percurso para a análise dos Microdados.

\footnotetext{
${ }^{12}$ Explicação dos cálculos (regra de três simples): aumento, conclusão final na discrepância da análise - Sinopse: 31.212 x 100/6.406 - 100 $($ aumento, portanto tiramos $100 \%$ da amostra inicial $)=387 \%$. Microdados: 51.652 x 100/8.808 $-100=486 \%$ ).
} 
A preocupação com a natureza desses bancos de dados problematiza a adequada utilização do censo escolar no que diz respeito a seus impactos na educação pública, mais especificamente nas ações necessárias para a identificação, atendimento e melhor distribuição e uso dos recursos financeiros, além, do fortalecimento de políticas públicas federais, estaduais e municipais já existentes e a formalização e regulamentação nos Estados mais carentes de legislações para as AHSD (BRASIL, 1996). Para que isso ocorra, faz-se necessário que os bancos de dados disponibilizem informações claras e, que no site do Inep elas sejam instrucionais e explicativas com orientações para análise dos Microdados, afinal, trata-se de resultados vinculados a Lei de Transparência e serem mais acessíveis.

Nessa perspectiva, Resende e Lacerda (2013) encontraram ambiguidades e imprecisões nos dados da Sinopse Estatística e do Microdados. Para elas, dados pouco explorados merecem ser analisados com a finalidade de visualizar a realidade frente à algumas variáveis, mas também, colaborar com tomadas de decisões e reflexões sobre os problemas educacionais, pois, os Microdados precisam ser investigados. Há um consenso entre as mencionadas autoras e Macalli et al. (2018) - trabalhar com Microdados requer um profundo conhecimento de análise estatística.

Embora possa haver limitações nos conhecimentos estatísticos, inclusive previstos, com base em outros autores, há discrepâncias nos resultados encontrados, na citada pesquisa, sobre o número total de matrículas. Os autores supracitados anteriormente, vale ressaltar, não comparam a Sinopse Estatística com os Microdados. Outro fator são os valores no que tange ao Estado de São Paulo, porque além do aumento em comparação aos outros Estados revela-se expressiva discrepância no banco Microdados nos anos de 2018 e 2019, conforme demonstrado na tabela 5.

Pelo exposto, alguns questionamentos mantêm-se sem respostas: essa discrepância nos valores ocorre por quê? Há um maior incentivo para a identificação no Estado de São Paulo? Por ter o Estado de São Paulo apresentado maior número de legislação pertinente à Educação Especial que contempla as AHSD? Será o impacto das atividades realizadas por pesquisadores da área das AHSD? Há um incentivo maior na formação de professores paulistas? Será problema ou equívoco nos registros dos cadastros dos estudantes? Ou o Estado de São Paulo conta com equipe educacional mais empenhada em identificar que em outros Estados?

Renzulli (2014; 2018; 2021), em suas pesquisas, assinala a importância da designação das AHSD e dos alunos serem visualizados pelos órgãos responsáveis pela Educação. Nos Estados Unidos, de acordo com o autor, sua teoria e modelo de enriquecimento foram aceitos quando seus resultados tornaram-se expressivos no desempenho educacional, ou seja, censo, avaliações externas, entre outros.

Diante dos resultados obtidos, nesta pesquisa, pode-se concluir que eles são seguramente significativos para serem compreendidos e estudados por quem de direito para que cumpram seu papel de servir à implementação de políticas públicas coerentes e eficazes ao público com AHSD. Nessa perspectiva, estudos que abordam e discutem os dados oficiais do censo escolar em especial para estudantes com AHSD, no Brasil, se fazem necessários inclusive que os remeta a outras regiões brasileiras para obtenção de um panorama do país.

\section{Agradecimentos}

Coordenação de Aperfeiçoamento de Pessoal de Nível Superior (Capes) - Código de financiamento 001.

\section{Referências}

Brasil. J. L. S. (2008). Instituto de Pesquisa Econômica Aplicada (Ipea). O que é IDH. (2008). https://www.ipea.gov.br/desafios/index.php?option=com_co ntent \&view=article\&id=2144: catid=28\&Itemid=23.

Colozio, A. R. S., Rangni, R. A. \& Borges, A. (2021). Altas Capacidades em meios Vulneráveis: produções acadêmicas brasileiras e internacionais. Research, Society and Development, 10(1), 01-13. Doi.10.33448/rsd-v10i1.11578.

Field, A. (2009). Descobrindo a estatística usando o SPSS (2a ed.). Artmed. 
Research, Society and Development, v. 10, n. 4, e7710413856, 2021

(CC BY 4.0) | ISSN 2525-3409 | DOI: http://dx.doi.org/10.33448/rsd-v10i4.13856

Instituto Nacional de Estudos e Pesquisas Educacionais Anísio Teixeira. (2020). http://inep.gov.br/web/guest/acesso-a-informacao

Instituto Brasileiro de Geografia e Estatística. Cidades e Estados. (2020). https://www.ibge.gov.br/cidades-e-estados/sp.html.

Lei n. 9.394, de 20 de dezembro de 1996 (1996). Estabelece as diretrizes e bases da educação nacional. Diário Oficial da Justiça. Brasília, DF: Presidência da República.

Koche, J. C. (2011). Fundamentos de metodologia científica: teoria da ciência e iniciação à pesquisa. Vozes.

Marconi, M. A. \& Lakatos, E. M. (2011). Técnicas de pesquisa (7a ed.). Atlas.

Macalli, A. C., Gonçalves, T. G. G. L. \& Caiado, K. R. M. (2018). Fluxo escolar de alunos com deficiência em um município: o que revelam os dados oficiais. Revista Educação Especial, 32, 1 - 20. 10.5902/198468628212.

Melletti, S. M. F. (2014). Indicadores Educacionais sobre a Educação Especial no Brasil e no Paraná. Revista Educação e Realidade, 39(3), 789-809. http://www.ufrgs.br/edu_realidade

Moreira, L. C. \& Carvalho, A. P. (2014). (Des) continuidade nos estudos de alunos com deficiência na trajetória do Ensino Fundamental ao Médio: uma análise inicial dos microdados MEC/INEP. Revista Educação Especial, 27(49), 283-298. doi.10.5902/1984686X9132.

Ministério da Educação. IDEB: apresentação. (2021). http://portal.mec.gov.br/conheca-o-ideb

Oliveira, L. P. \& Rangni, R. A. (2019). Sinopse estatística da educação básica: o lugar dos alunos com alta capacidade. Revisa Poiésis, 13(23), 480-496. doi.10.0.74.233/prppge.v13e242019480-496.

Pereira, A. S., Shitsuka, D. M., Pareira, F. J. \& Shitsuka, R. (2018). Metodologia da pesquisa científica. UFSM.

Renzulli, J. S. (2014). A concepção de superdotação no modelo dos três anéis: um modelo de desenvolvimento para a promoção da produtividade criativa. In: A. Virgolim \& E. C. Konkiewitz (Orgs.), Altas Habilidades/Superdotação: inteligência e criatividade (p. 219 - 264). Papirus.

Renzulli, J. S. (2018). Reexaminando o papel da educação para superdotados e o desenvolvimento de talentos para o século XXI: uma abordagem teórica em quatro partes. In: A. Virgolim (Org.), Altas Habilidades/Superdotação: processos criativos, afetivos e desenvolvimento de potenciais (p. 19 - 42).

Renzulli, J. S. (2021). Reflections on my work: the identification and development of creative of creative/productive giftedness. In D. Y. Dai \& R. J. Sternberg (Orgs.), Scientific Inquiry into human potencial: historical and contemporary perspectives acress disciplines (p.197-211).

Resolução $n^{\circ}$ 4, de 02 de outubro de 2009. Institui Diretrizes Operacionais para o Atendimento Educacional Especializado na Educação Básica, modalidade Educação Especial. Brasília, DF: Diário Oficial da União, 05 out. 2009. Seção 1, 17-17. https://www.jusbrasil.com.br/diarios/897119/pg-17-secao-1-diariooficial-da-uniao-dou-de-05-10-2009.

Resende, A. A. C. \& Lacerda, C. B. F. (2013). Mapeamento de Alunos Surdos Matriculados na Rede de Ensino Pública de um Município de Médio Porte do Estado de São Paulo: Dissonâncias. Revista brasileira de Educação Especial, 19(3), 411-424. doi.org/10.1590/S1413-65382007000300009

Rebelo, A. S. \& Kassar, M. C. M. (2018). Escolarização dos alunos da educação especial na política de educação inclusiva no Brasil. Revista inclusão social, 11(01), 56-66. http://revista.ibict.br/inclusao

Sales, L. C. \& Silva, M. J. G. S. (2013). A Movimentação das Matrículas no Ensino Fundamental no Estado do Piauí. Revista Educação e Realidade, 38(04), 1283-1301. http://www.ufrgs.br/edu_realidade

Santos, V. \& Elias, N. C. Characterization of enrollments of students with autism spectrum disorders by brazilian regions. Revista brasileira de Educação Especial. 24(4), 459-476. https://doi.org/10.1590/s1413-65382418000500001

Senado Federal. (2020). https://www.camara.leg.br/noticias/714845-camara-realiza-sessao-para-votar-regras-do-fundeb/ 